\title{
MIXMASTER CHAOS VIA THE INVARIANT MEASURE
}

\author{
GIOVANNI IMPONENTE \\ Dipartimento di Fisica, Universitá "Federico II", Napoli and INFN - Napoli - Italy \\ ICRA - International Center for Relativistic Astrophysics \\ E-mail: imponente@icra.it \\ GIOVANNI MONTANI \\ Dipartimento di Fisica - G9 Universitá "La Sapienza", Roma - Italy \\ ICRA - International Center for Relativistic Astrophysics \\ E-mail: montani@icra.it
}

\begin{abstract}
The chaoticity of the Mixmaster is discussed in the framework of Statistical Mechanics by using Misner-Chitre-like variables and an ADM reduction of its dynamics.

We show that such a system is well described by a microcanonical ensemble whose invariant measure is induced by the corresponding Liouville one and is uniform. The covariance with respect to the choice of the temporal gauge of the obtained invariant measure is outlined.
\end{abstract}

\section{Introduction}

The original treatment of the Mixmaster 1 chaoticity due to Belinski, Khalatnikov and Lifshitz (BKL) 2 provided a satisfactory description of the Kasner indices behaviour, especially because the invariant measure for the Poincare return map was calculated in terms of the $u$ parameter. Nevertheless the question about how to construct a presentation for the system stochasticity in terms of continuous variables remained open. Relevant achievements in this direction appeared in 345, where an invariant measure associated to the motion of the billiard ball representing the system is studied in terms of continuous Misner-Chitre-like variables.

Here we review the fundamental steps at the grounds of this analysis and focus our attention to the invariance of the statistical mechanics representation with respect to the choice of the lapse function $[5$.

\section{Asymptotic Dynamics}

Near the cosmological singularity the Mixmaster model admits a dynamics described by the two-dimensional ADM reduced action

$$
\mathcal{S}_{\mathrm{RED}}=\int_{\Gamma_{H}}\left(p_{\xi} d \xi+p_{\theta} d \theta-\varepsilon d f\right)
$$


being

$$
\varepsilon^{2}=\left(\xi^{2}-1\right) p_{\xi}^{2}+\frac{p_{\theta}^{2}}{\xi^{2}-1}
$$

we adopted Misner-Chitre-like variables $\xi, \theta, f(\tau) \underline{6}$ and $p_{\xi}$ and $p_{\theta}$ denote the conjugate momenta to $\xi$ and $\theta$, respectively.

This picture is associated to the temporal gauge

$$
N(\tau)=\frac{12 D}{E} e^{2 f} \frac{d f}{d \tau}
$$

in which $D=\exp \left(-3 \xi e^{f(\tau)}\right)$ and $E$ denotes a generic positive value for the energylike constant of motion $\varepsilon$ and the fixing of a specific time variable corresponds to choose a suitable function $f(\tau)$. The integral in (1) is referred to the domain $\Gamma_{H}$ (see Figure 1) outside of which the motion is classically forbidden; $\Gamma_{H}$ is dynamically closed and corresponds to a portion of a two-dimensional Lobachevsky plane, i.e. a surface of constant negative curvature.

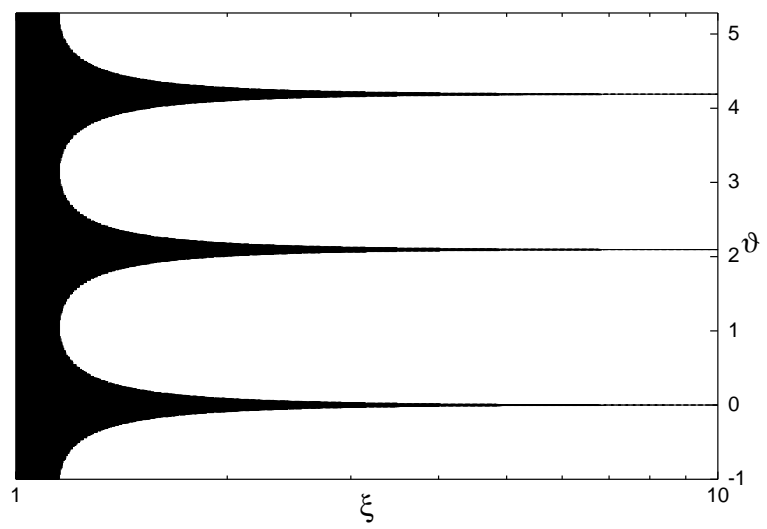

Figure 1. Domain $\Gamma_{H}$ dynamically accessible

\section{Invariant Measure}

This representation of the Mixmaster dynamics in terms of a billiard ball on a Lobachevsky plane is manifestly chaotic and the existence of an energy-like constant of motion characterizes the statistical properties by a microcanonical ensemble; therefore the distribution function takes the Liouville form

$$
d \mu \propto \delta(E-\varepsilon) d \xi d \theta d p_{\xi} d p_{\theta} .
$$


Since the value of $\varepsilon$ cannot contain any information about the Mixmaster chaoticity then we have to integrate on this variable by redefining the momenta as follows

$$
\begin{aligned}
& p_{\xi}=\frac{\varepsilon}{\sqrt{\xi^{2}-1}} \cos \phi \\
& p_{\theta}=\varepsilon \sqrt{\xi^{2}-1} \sin \phi,
\end{aligned}
$$

where $0 \leq \phi<2 \pi$; hence we get an uniform invariant measure as written in the variables $\xi, \theta, \phi$ (in the phase space $\varepsilon$ assumes a fixed value), i.e.

$$
w_{\infty}=\left\{\begin{array}{lc}
\frac{1}{8 \pi^{2}} & \text { in } \Gamma_{H} \\
0 & \text { outside } \Gamma_{H}
\end{array}\right.
$$

The invariant measure (6) holds in the time variable $f$ and can be taken as the stationary solution of the Liouville equation

$$
\begin{aligned}
\frac{d w}{d f}=\frac{\partial w}{\partial f}+\frac{d \xi}{d f} \frac{\partial w}{\partial \xi} & +\frac{d \theta}{d f} \frac{\partial w}{\partial \theta}+\frac{d \phi}{d f} \frac{\partial w}{\partial \phi}= \\
& =\frac{\partial w}{\partial f}+\sqrt{\xi^{2}-1} \cos \phi \frac{\partial w}{\partial \xi}+\frac{\sin \phi}{\sqrt{\xi^{2}-1}} \frac{\partial w}{\partial \theta}-\frac{\xi \sin \phi}{\sqrt{\xi^{2}-1}} \frac{\partial w}{\partial \phi}=0
\end{aligned}
$$

associated to the Hamiltonian system

$$
\frac{d \xi}{d f}=\sqrt{\xi^{2}-1} \cos \phi, \quad \frac{d \theta}{d f}=\frac{\sin \phi}{\sqrt{\xi^{2}-1}}, \quad \frac{d \phi}{d f}=-\frac{\xi \sin \phi}{\sqrt{\xi^{2}-1}} .
$$

The invariance of distribution (66) is ensured by the holding of Eq. (7) in any other time variable; in fact, the time gauge (3) allows to re-express the Liouville theorem (17) in a generic variable $\tau$ via a simple multiplication by a common factor.

Indeed for our proof is relevant that we are dealing with a stationary distribution function; for non-stationary corrections to the measure (6) and the corresponding asymptotic behaviour of the right-hand side of (7) see 17. However, all such nonstationary terms decay exponentially and do not affect the results here presented.

\section{References}

1. C.W. Misner (1969) Phys. Rev. Lett., 22, 1071.

2. V.A. Belinski, I.M. Khalatnikov and E.M. Lifshitz (1970) Adv. Phys. 19, 525.

3. D.F. Chernoff and J.D. Barrow (1983) Phys.Rev.Lett. 50134.

4. A.A. Kirillov and G. Montani (1997) Phys.Rev. D 56, 6225.

5. G.P. Imponente and G. Montani (2002) Int.Journ.Mod.Phys.D 11 n.8, 1321.

6. G.P. Imponente and G. Montani (2001) Phys.Rev. D 63, 103501.

7. G. Montani (2001) Il Nuovo Cimento 116 B n.12, 1375. 